\title{
Application of computational chemistry in chemical reactivity: a review
}

\author{
C. W. Chidiebere ${ }^{\mathrm{a}, *}$, C. E. Duru ${ }^{\mathrm{a}}$, JP. C. Mbagwu ${ }^{\mathrm{b}}$ \\ ${ }^{a}$ Department of Chemistry, Imo State University, Surface Chemistry and Environmental Technology (SCENT) Research Unit, Department of Chemistry, Imo State \\ University, Owerri, Imo State, Nigeria \\ ${ }^{b}$ Department of Physics, Faculty of Physical Sciences, Imo State University, Owerri, Nigeria.
}

\begin{abstract}
Molecular orbitals are vital to giving reasons several chemical reactions occur. Although, Fukui and coworkers were able to propose a postulate which shows that highest occupied molecular orbital (HOMO) and lowest unoccupied molecular orbital (LUMO) is incredibly important in predicting chemical reactions. It should be kept in mind that this postulate could be a rigorous one therefore it requires an awfully serious attention in order to be understood. However, there has been an excellent breakthrough since the introduction of computational chemistry which is mostly used when a mathematical method is fully well built that it is automated for effectuation and intrinsically can predict chemical reactivity. At the cause of this review, we've reported on how HOMO and LUMO molecular orbitals may be employed in predicting a chemical change by the utilization of an automatic data processing (ADP) system through the utilization of quantum physics approximations.
\end{abstract}

DOI:10.46481/jnsps.2021.347

Keywords: HOMO-LUMO, Computational methods, and Chemical reactivity

Article History :

Received: 14 August 2021

Received in revised form: 30 September 2021

Accepted for publication: 01 October 2021

Published: 29 November 2021

(C)2021 Journal of the Nigerian Society of Physical Sciences. All rights reserved. Communicated by: E. A. Emile

\section{Introduction}

Frontier molecular orbital theory was postulated in 1952 by Kenichi Fukui where he described highest molecular orbital (HOMO) and lowest unoccupied molecular orbital (LUMO) interaction from molecular orbital theory [1]. In 20th century, electronic structure of a molecule was described using molecular orbital theory from quantum physics [2], where electrons are treated as moving under the control of the atomic nucleus within the bulk molecule but aren't treated as a personal attraction between atoms in line with molecular orbital theory.

\footnotetext{
${ }^{*}$ Corresponding author tel. no: +234

Email address: oraclewizzy@gmail.com (C. W. Chidiebere)
}

The reason of the spacing of electrons was given by quantum physics. However, quantum physics also explains the energetic behaviour of electrons as molecular orbital which house two or more atoms $[3,4]$. There's little question, that molecular orbital theory has changed how chemical bonding is viewed through the approximation of the states of bonded electrons i.e., viewing the molecular orbital as linear combinations of atomic orbital (LCAO) [5]. These approximations are made by applying the density functional theory (DFT) model or HartreeFork (HF) models to the time independent differential equation. These approximations are the fundamental theories in computational chemistry [6]. They'll be modeled and automatic in a very automatic data processing system to help easy calculation. However, computational chemistry may be utilized in different 
number of ways but the foremost important one is to model a molecular system by synthesizing the molecule in a laboratory [7]. Confine in mind that computational models don't seem to be always perfect but it can rule out about $90 \%$ of possible molecules that don't seem to be good for his or her intended use [8], however, computational chemistry makes it easy to understanding a controversy fine.

In the study of chemical reactivity, both state and open system (involving radicals, cations, and anions) calculations are performed to establish a result [9]. These methods are frequently unreliable, especially when diffuse functions must be included in the basis set. As a result, it is useful to have a technique that can extract all of the information required directly from the findings of the molecular system's basis state calculation [10]. Thus, the knowledge of frontier molecular orbitals has imparted on the understanding of chemical reactions and is accustomed to distinguish between the parts of orbital that have opposite signs.

\section{Highest Occupied and Lowest Unoccupied Molecular Or- bital (HOMO-LUMO)}

Highest occupied molecular orbital (HOMO) is that outermost orbital where the very best energy orbital is contained, the orbital acts as an electron donor while lowest unoccupied molecular orbital (LUMO) is that orbital with the lowest unoccupied energy level which acts as the electron receiver. According to the frontier molecular orbital theory, the formation of a transition state is because of a synergy between the frontier orbitals of the reactants [11]. The stability of a molecule is determined by HOMO-LUMO gap which is known as the dissimilarity in energy between the HOMO and LUMO of the molecule [12]; thus, a large HOMO-LUMO gap implies high stability for the molecule in chemical reactions while a tiny low one implies low stability. Thus, the HOMO-LUMO gap may be defined mathematically as [13]:

$$
\Delta E=E_{L U M O}-E_{\text {HOMO }}
$$

where $\Delta E$ is the Energy gap, $E_{L U M O}$ is the Energy of LUMO and $E_{\text {Номо }}$ is the Energy of HOMO. Keep in mind that when the HOMO-LUMO gap is large, the molecule is said to be hard and a soft molecule has a small energy gap [14]. Thus, chemical hardness is the opposition to change in electron cloud density or electron distribution of a chemical system and chemical softness is the inverse of chemical hardness [15]. Chemical hardness and chemical softness can be shown mathematically as

$$
\begin{aligned}
& \eta=\frac{I-E A}{2} \\
& \sigma=\frac{1}{\eta}
\end{aligned}
$$

where $\eta$ is known as chemical hardness, $\sigma$ is known as chemical softness.
Ionization potential relates to energy of the HOMO while the energy of the LUMO relates to the electron affinity thus the HOMO and LUMO energies can be applied in the calculation of the ionization potential (I) and electron affinity EA. From Koopmans theorem [16]

$$
\begin{aligned}
& I=-E_{H} \\
& E A=-E_{L}
\end{aligned}
$$

\section{Density Functional Theory (DFT)}

Density functional theory also referred chemical reactivity theory is a variational method that's currently the foremost promising approach which is employed to determining the electronic structure of matter [17]. The N-particle differential equation yields density functional theory, which is totally stated in terms of the density distribution of the base state and the single particle wave function. It's been a helpful tool which is employed in analysis, prediction and elucidation of the results of chemical reactions. Following the work of Parr et al [18], advantageous number of ideas has been derived from the resolution of the density of molecular systems using DFT. its requisite to own recourse on the Kohn-Sham theory by calculating the molecular density, the system's energy and energies of the orbital especially; those connected to the frontier orbitals. The kohn-Sham method creates a precise connection between the density and state energy of a non-interacting fermion system and the real many bodies system given by the Schrodinger wave equation [19].

$$
\frac{-\hbar}{2 m} \frac{\partial^{2}}{\partial x^{2}}=i \hbar \frac{\partial \Psi}{\partial t}
$$

The basis of DFT has confirmed the existence of a universal density functional, and the system's properties are acquired by completing calculations using the functionals [20]. Because they're approximate functionals rather than universal functionals, some of them are good at predicting certain qualities while others are good at predicting others. Density functionals that are excellent at defining the features of each molecule system with a specific functional group can occasionally be discovered. For the study of molecular systems, it's quite advantageous to employ different density functionals for a separate functional group that you merely want to require in [21]. DFT reduces the time it takes to compute the bottom state parameters of systems of synergizing particles to the same precision as the results of single-particle Hartree-type equations, which is why it's so beneficial for systems with many electrons. The goal of DFT calculations is to show how the electron density is related to the bottom state. By using the results of the Kohn-Sham equations, the definition of electron density is given [22].

$$
\rho(r)=\sum_{i} \Psi_{i}(r) \Psi_{i}^{*}(r)
$$



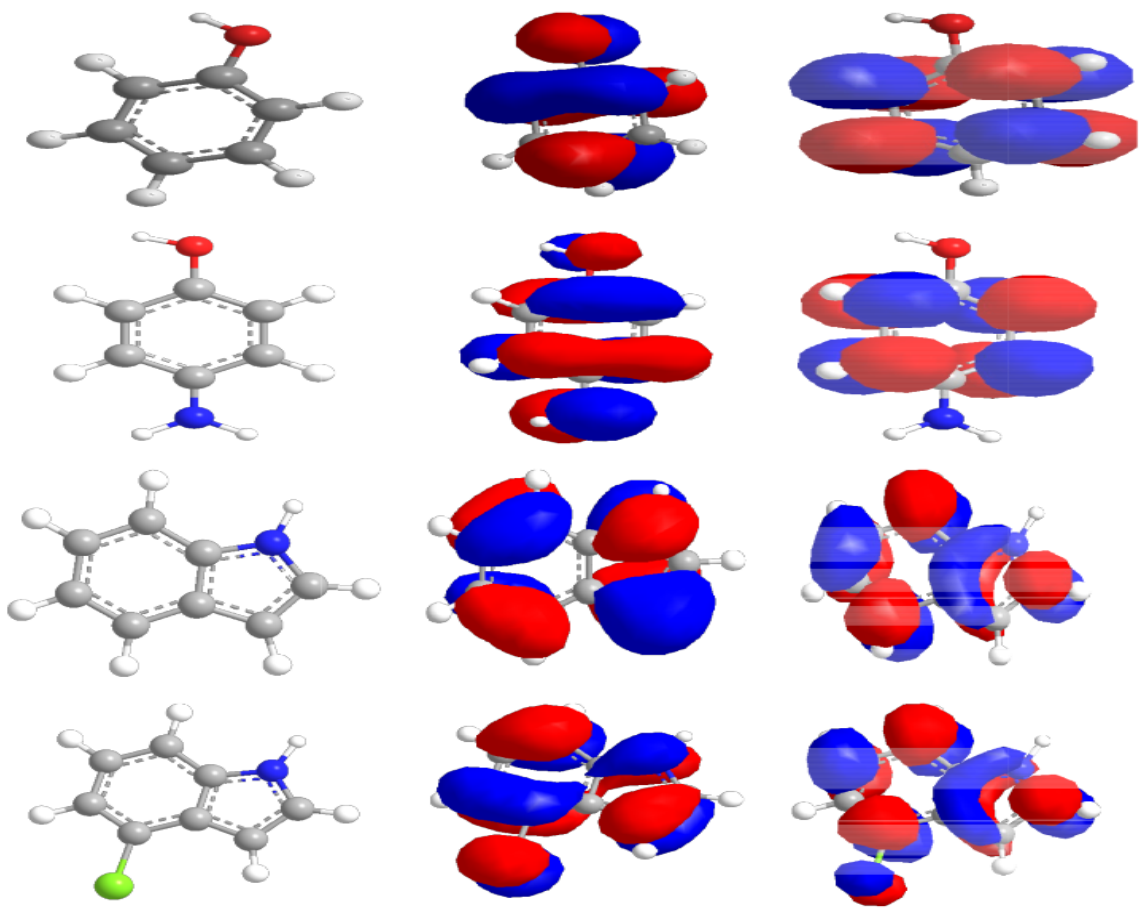

Figure 1: Aromatic compounds with the highest occupied molecular orbital (HOMO) and the lowest unoccupied molecular orbital (LUMO) were discovered using DFT.

\section{Hatree-Fock Equation}

The Hartree-Fork Fork technique is an approximation approach used in computational chemistry to derive the wave function with the energy of a quantum body system in an immobile mmobile condition [23]. Hartree-Fork theory is a fundamental notion in electronic structure theory; it is the cornerstone of molecular orbital theory, which states that each electron's mobility may be described by a single-particle particle function orbital that is independent of the other electrons'immediate motion [24].The Hartree-Fork resolves the set of spin orbitals which reduce the energy and devote the best single determinant. The equation is given as [25].

$$
\begin{aligned}
h\left(X_{1}\right) \chi_{i}\left(X_{1}\right) & +h \sum_{j \neq i}\left[\int d x_{2}\left|x_{j}\left(X^{-1}\right)\right|^{2} r_{12}^{-1}\right] \chi_{i}\left(X^{-1}\right) \\
& -\sum_{j \neq i}\left[d x_{2} \chi_{j}^{*}\left(X^{2}\right) \chi_{i}\left(X^{2}\right) r_{12}^{-1} x_{j}\left(X^{1}\right)=\epsilon_{i} \chi_{i}\left(X_{1}\right)\right],
\end{aligned}
$$

where $\epsilon_{i}$ is the energy eigenvalue associated with orbital $\left(\chi_{i}\right)$. Hence the Hartree-Fork equations can be resolved mathematically i.e., exact Hartree-Fork, or can be solved in the spaced spanned by a set of fundamental functions (Hartree-Fork-Roothan equations) where their solutions are dependent on the orbitals [26].
Reducing Hartree-Fork equation to Hartree-Fork-Roothan equation in matrix form, we have:

$$
\sum_{v} F_{\mu \nu} C_{v i}=\epsilon_{i} \sum_{v} S_{\mu \nu} C_{v i}
$$

\section{Frontier molecular orbital calculation and application of computer system in computational chemistry}

Calculation of molecular orbital energy may be done using ab initio methods; the tactic applies electron and also the nuclei synergy as its fundamental component. It attempts to calculate the electronic differential equation by assigning the locations of the nuclei and therefore the electrons surrounding it. Hence, electronic energy of a molecule may be calculated by treating the general electronic wave function as being a product of molecular orbitals which is predicated on Slater determinant of molecular orbitals so as to satisfy the Pauli exclusive principle [27]. So, we will write a molecular wave function as:

$$
\Psi=\phi^{1} \phi^{2} \phi^{3} \phi^{4} \cdots \phi^{n}
$$

where each molecular orbital is written in form of a linear combination of atomic orbitals that is,

$$
\Psi_{i}=\sum_{i} c_{i} k \Phi
$$

The molecular orbitals will be calculated by using the HartreeFock method [28]. The Hamiltonian operator has terms for the 
potential and mechanical energy of the electrons; it involves the interactions between the nuclei and the electrons, and also the electron-electron repulsion. Note the molecular orbitals are conceived by making an initial supposition at the coefficients [29]. Hence

$$
H-E S=0
$$

$H$ contains the Hamiltonian matrix elements and $S$ is known as the overlap matrix elements. Solving this determinant will give the answer of molecular orbital energy $E$, which is substituted into the overall equations to allow new values of the coefficients $\left(c_{i}\right)$. This whole process is repeated until the coefficients don't change from one cycle to the other. The orbitals can then be described as self-consistent, and this process is thought as a selfconsistent field procedure [30] where each molecular orbital is created from a mixture of atomic orbitals.

When one function is employed for every filled atomic orbital for instance, using, an orbital for an atom, this basis set is termed a minimal basis [31]. Using more functions than this may improve the calculation i.e., it'll tend to extend the pliability and quality of the general wave function. From the variational principle we all know that a stronger wave function will provides a lower energy [32]. The larger the idea set the higher the calculation, if a large number of functions i.e., a really large basis set is employed to point out the atomic orbitals; we approach the Hartree-Fock limiting energy. However, a very cheap energy is achieved by the Hartree-Fock method [33].

Frontier molecular orbital theory is an exemplification of molecular orbital theory which elucidates HOMO/LUMO synergies [34]. During 1952, Kenichi Fukui put forth a paper within the Journal of Chemical Physics which he titled "A molecular theory of reactivity in aromatic hydrocarbons". His work examined the frontier orbitals and additionally the consequences of the HOMO and also the LUMO on reaction mechanism specifically which is the reason why it's called frontier molecular orbital theory (FMOT) [35]. Fukui noticed that an honest approximation for reactivity can be established by observing the HOMO-LUMO. This was founded on principal point observance of molecular orbital theory of two molecules which shows that the occupied orbital of unlike molecules opposes one another, thus, the negative charges of a molecule attract positive charges of another molecule as such, the occupied orbital of another molecule and the unoccupied orbital of the opposite synergize with one another per se causing attraction [36].

Though this theory could also be a rigorous narrative of chemical phenomena, impediment within the mathematical aspect may well be too difficult that it'll not be viable to resolve a controversy accurately. Since the introduction of computational chemistry, most rigorous theories can now be treated with little difficulty. Computational chemistry is employed in a very number of various ways, one in all which is to model a molecular system before synthesizing the molecule within the laboratory [37]. However computational models don't seem to be always perfect but it can rule out about $90 \%$ of possible molecules that don't seem to be good for its intended use [38].

However, it is very helpful because to synthesizing only one (1) compound could take months of labour and raw materials and furthermore generate waste matter. Computational chemistry helps in comprehending an issue wholly. There are some properties of a molecule that may be gotten computationally more easily than by experimental means [39]. There also are deep views into molecular bonding, which might be gotten from the inference of computations that can't be obtained from any scientific method. Thus, many experimental chemists are now making use of computational modeling to induce additional understanding of the compounds being observed within the laboratory [40].

Going back to history, Paul Cruzan, Mario Molina, and Sherwood Rowland who are computational chemists were awarded the laurels in chemistry in 1995 for forming mathematical models that applied the thermodynamic and chemical laws to elucidate how ozone were formed and decomposed within the atmosphere [42]. Although, computational chemistry wasn't usually imagined as its own separate field of study until 1998, when Walter Kohn and John Pople were awarded the Nobel prize in Chemistry for their exhibition of density functional theory and disclosure of computational methods using quantum chemistry respectively [43].

Computational chemistry is not the same as engineering science; however professionals from both fields frequently collaborate. Computer scientists spend the majority of their work assembling and testing computer algorithms, data visualization skills, and software and hardware solutions [44].Computational chemists work alongside laboratory scientists and theoretical scientists to put all of this modeling, simulation, data analysis, and visualization expertise to good use in their study. Computational chemists can employ supercomputers and computing clusters, which are high-performance computing systems, to solve issues and run simulations that require a large quantity of data [45]. Electronic structure approaches, molecular dynamics simulations, quantitative structure-activity connections, and complete statistical analysis are among the tools used by computational chemists.

To combine chemical theory and modeling with actual observations, computational chemists use statistics, mathematical algorithms, and massive databases. Some computational chemists create physical step models and simulations, and then utilize statistics and data analysis approaches to extract useful data [46]. Progress in computer visualization allows computational chemists to highlight complex analyses in a more comprehensive format, which they may use to explain experiments and current materials, as well as validate the results.

Computational chemistry advances our understanding of how the world works, aids manufacturers in developing more efficient and productive operations, allows for the characterization of new chemicals and materials, and aids other researchers in extracting relevant knowledge from large amounts of data. 


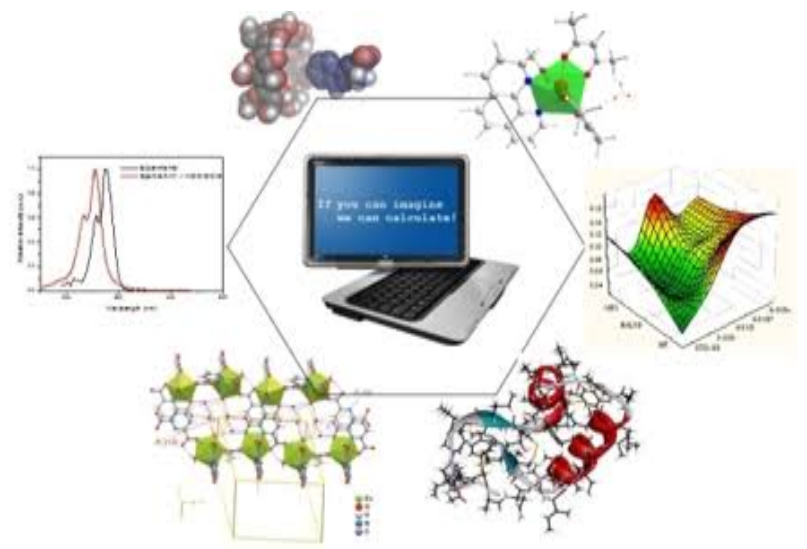

Figure 2: Shows the aid of computer system in elucidation of chemical behaviour

Quantum physics and thermodynamics are used in computational chemistry to learn about the character and fundamental properties of atoms, molecules, and chemical reactions [47].

Recent researches showed that quantum physics $(\mathrm{QM})$ and molecular mechanics are the foremost used models in computation. Most computation software's utilized by researchers include:

\section{- Integrated packages}

- ab initio and DFT software

- Semi empirical software

- Graphics package

- Special purpose program

- Molecular mechanics/molecular dynamics/Monte Carlo software

Software packages differ in cost, functionality, efficiency, automation, robustness and ease to use. These concerns make a difference in determining what computational projects is successful and the way much work which will be involved [48].

\section{Conclusion}

The quest for understanding chemical reaction mechanism has really caused many researchers to go into in-depth study to source out path of reactions and the cause of the reactions. HOMO-LUMO molecular orbitals have changed the perspective at which chemists view chemical reactions with the help of quantum mechanics which has given the exact explanation on the behaviour of electrons. Apart from this, scientists went further to develop models from quantum mechanics approximations of molecular orbital theory. These approximations were achieved by applying Hartree-Fork and density theory functionals which are approximations to determine a many electrons body system. A further study on the variational methods can improve research work on computational chemistry thus giving a more easy and reliable way for solving problems.

\section{References}

[1] L. S. Braga, D. H. Leal, K. Kuca, \& T. C. Ramalho, "Perspectives on the Role of the Frontier Effective-for-Reaction Molecular Orbital (FERMO) in the Study of Chemical Reactivity", An Updated Review Current Organic Chemistry 24 (2020) 314.

[2] D. S.Taylor \& A. Holewinski, "Selective Interactions between FreeAtom-like d-States in Single-Atom Alloy Catalysts and Near-Frontier Molecular Orbitals", Journal of the American Chemical Society 13 (2021) 342.

[3] F. Guohong, Q. Wang, X. Hong, X. Wang, T. Xianxian, \& C. Xiangfeng, "Single $\mathrm{Cr}$ atom supported on boron nitride nanotubes for the reaction of $\mathrm{N} 2 \mathrm{O}$ reduction by CO: A density functional theory study", Applied Surface Science 544 (2021) 148776.

[4] F. Wang, L. Zhongling, L. Yan, S. Alessandro, J. M. Poblet, \& C. de Graaf, "Density functional theory study of single-molecule ferroelectricity in Preyssler-type polyoxometalates", APL Materials 9 (2021) 021109.

[5] X. Wei, M. Miaomiao, H. Zhang, L. Wang, \& C. Dong, "Density functional theory study of ultra-thin In Se electrodes for $\mathrm{Na}$ and $\mathrm{Mg}$ ion storage and transport”, Materials Letters 285 (2021) 129091.

[6] M. Itakura, M. Yamaguchi, D. Egusa, \& E. Abe, "Density functional theory study of solute cluster growth processes in Mg-Y-Zn LPSO alloys", Acta Materialia 203 (2021) 116491

[7] L. Shi, Y. Huang, L. Zhang-Hui, C. Wanglai, Y. Xiaohu, S. Qing, Z. Gao, R. Zhang, \& G. Feng, "Surface property of the $\mathrm{Cu}$ doped $\gamma$-Al2O3: A density functional theory study" Applied Surface Science 535 (2021) 147651.

[8] P. Chen, G. Mingyan, D. Wang, J. Wang, X. Huang, H. Wang, \& Y. Lin, "Experimental and density functional theory study of the influence mechanism of oxygen on NO heterogeneous reduction in deep air-staged combustion", Combustion and Flame 223 (2021) 127.

[9] K. Park, J. Byeong-Hyeon, H. Y. Lim, \& P. Ji-Sang, "Effect of chemical substitution on polytypes and extended defects in chalcopyrites: A density functional theory study", Journal of Applied Physics 129 (2021) 025703 .

[10] B. B. Xiao, L. Yang, H. Y. Liu, X. B. Jiang, B. Aleksandr, E. H. Song, \& Q. Jiang, "Designing fluorographene with FeN4 and CoN4 moieties for oxygen electrode reaction: A density functional theory study", Applied Surface Science 537 (2021) 147846.

[11] A. Liu, W. Guan, W. Kefan, X. Ren, L. Gao, \& M. Tingli, "Density functional theory study of nitrogen-doped graphene as a high-performance electrocatalyst for CO2RR”, Applied Surface Science 540 (2021) 148319.

[12] Y. Yang, A. Sun, \& M. Eslami, "A density functional theory study on detection of amphetamine drug by silicon carbide nanotubes", Physica E: Low-dimensional Systems and Nanostructures 125 (2021) 114411.

[13] R. Hussain, S. Muhammad, M. Y. Mehboob, S. U. Khan, M. U. Khan, M. Adnan, M. Ahmed, J. Iqbal, \& K. Ayub, "Density functional theory study of palladium cluster adsorption on a graphene support", RSC Advances 10 (2020) 20595.

[14] A. S. Shakeel \& D. C. Gupta, "Investigation of structural, elastic, thermophysical, magneto-electronic, and transport properties of newly tailored Mn-based Heuslers: A density functional theory study", International Journal of Quantum Chemistry 120 (2020) 26216.

[15] Z. Jafari, R. Baharfar, A. R. Shokuhi, \& S. Asghari, "Potential of graphene oxide as a drug delivery system for Sumatriptan: a detailed density functional theory study", Journal of Biomolecular Structure and Dynamics 39 (2020) 1.

[16] J. Keunhong, H. J. Jeong, L. Gunwoo, S. H. Kim, H. K. Kwang, \& G. Y. Chang, "Catalytic effect of alkali and alkaline earth metals in lignin pyrolysis: A density functional theory study", Energy \& Fuels 34 (2020) 9734.

[17] P. Zhao, B. Boekfa, S. Ken-ichi, M. Ogura, \& M. Ehara, "Selective catalytic reduction of $\mathrm{NO}$ with $\mathrm{NH} 3$ over $\mathrm{Cu}$-exchanged $\mathrm{CHA}, \mathrm{GME}$, and AFX zeolites: a density functional theory study", Catalysis Science \& Technology 11 (2021) 1780.

[18] C. Yuan, C. Zhang, Y. Shenghui, H. Xu, L. Xin, Q. Fang, \& G. Chen, "Experimental and Density Functional Theory Study of the Adsorption Characteristics of $\mathrm{CaO}$ for $\mathrm{SeO} 2$ in Simulated Flue Gas and the Effect of CO2", Energy \& Fuels 34 (2020) 10872.

[19] M. Masoumi, M. Jahanshahi, M. G. Ahangari, G. \& N. Darzi, "Density functional theory study on the interaction of chitosan monomer with 
$\mathrm{TiO}_{2}, \mathrm{SiO}_{2}$ and carbon nanotubes", Materials Chemistry and Physics 255 (2020) 123 .

[20] Z. Lyu, S. Niu, L. Chunmei, G. Zhao, Z. Gong, \& Y. Zhu, "A density functional theory study on the selective catalytic reduction of NO by NH3 reactivity of $\alpha$ - $\mathrm{Fe}_{2} \mathrm{O}_{3}\left(\begin{array}{lll}0 & 0 & 1\end{array}\right)$ catalyst doped by $\mathrm{Mn}, \mathrm{Ti}, \mathrm{Cr}$ and Ni", Fuel 267 (2020) 117.

[21] O. Zhiliang, R. Jingyu, N. Juntian, Q. Changlei, H. Wei, \& L. Yang, "A density functional theory study of $\mathrm{CO} 2$ hydrogenation to methanol over $\mathrm{Pd} / \mathrm{TiO} 2$ catalyst: The role of interfacial site", International Journal of Hydrogen Energy 45 (2020) 6328.

[22] A. A. Peyghan \& J. Beheshtian, "Application of hexa-perihexabenzocoronene nanographene and its $\mathrm{B}, \mathrm{N}$, and $\mathrm{Bn}$ doped forms in Na-ion batteries: A density functional theory study", Thin Solid Films 704 (2020) 137.

[23] H. Li, Z. Zhang, Y. Liu, C. Wanglai, \& X. Luo, "Functional group effects on the HOMO-LUMO gap of g-C3N4 Nanomaterials" 8 (2018) 589,

[24] S. R. Wang, M. Arrowsmith, J. Böhnke, H. Braunschweig, T. Dellermann, R. D. Dewhurst, H. Kelch, I. Krummenacher, J. D. Mattock, J. H. Müssig, T. Thiess, A. Vargas, \& J. Zhang, "Engineering a Small HOMO-LUMO Gap and Intramolecular C-H Borylation by Diborene/Anthracene Orbital Intercalation", Angewandte Chemie International Edition 56 (2017) 8009.

[25] C. Dongping \& H. Wang, "HOMO-LUMO energy splitting in polycyclic aromatic hydrocarbons and their derivatives", Proceedings of the Combustion Institute 37 (2019) 953.

[26] Y. Huang, C. Rong, R. Zhang, \& S. Liu, "Evaluating frontier orbital energy and HOMO/LUMO gap with descriptors from density functional reactivity theory", Journal of molecular modeling 23 (2017) 1.

[27] Y. Morisawa, S. Tachibana, A. Ikehata, T. Yang, M. Ehara, \& Y. Ozaki, "Changes in the Electronic States of Low-Temperature Solid nTetradecane: Decrease in the HOMO-LUMO Gap", ACS omega 2 (2017) 618.

[28] D. Chen \& H. Wang, "Homo-lumo gaps of homogeneous polycyclic aromatic hydrocarbon clusters", The Journal of Physical Chemistry 123 (2019) 27785.

[29] R. Gershoni-Poranne, A. P. Rahalkar, \& A. Stanger, "The predictive power of aromaticity: quantitative correlation between aromaticity and ionization potentials and HOMO-LUMO gaps in oligomers of benzene, pyrrole, furan, and ...", Physical Chemistry Chemical Physics 20 (2018) 14808.

[30] A. B. Marahatta, "Computational Study on Electronic Structure, Atomic Charges Distribution and Frontier Molecular Orbitals of Butadiene: General Features for Diels-Alder Reaction", International Journal of Progressive Sciences and Technologies 19 (2020) 48.

[31] F. A. Bulat, J. S. Murray, \& P. Politzer, "Identifying the most energetic electrons in a molecule: The highest occupied molecular orbital and the average local ionization energy", Computational and Theoretical Chemistry 1199 (2021) 113192.

[32] P. Vinduja, V. K. Rajan, S. Krishna, \& K. Muraleedharan, "A Computational Modeling of the Structure, Frontier Molecular Orbital (FMO) Analysis, and Global and Local Reactive Descriptors of a Phytochemical 'Coumestrol' ", Mathematics Applied to Engineering in Action (2021) 41.

[33] K. Harismah, A. M. Dhumad, H. S. Ibraheem, H. Zandi, \& H. J. Ma- jeed, "A DFT approach on tioguanine: Exploring tio-tiol tautomers, frontier molecular orbitals, IR and UV spectra, and quadrupole coupling constants", Journal of Molecular Liquids 334 (2021) 116018.

[34] D. Baelde, S. Delaune, C. Jacomme, A. Koutsos, \& S. Moreau, "An interactive prover for protocol verification in the computational model", SP 2021-42nd IEEE Symposium on Security and Privacy.

[35] E. M. Heffernan, J. D. Adema, \& M. L. Mack, "Identifying the neural dynamics of category decisions with computational model-based functional magnetic resonance imaging", Psychonomic Bulletin \& Review (2021) 1.

[36] M. Abadi, M. Isard, \& D. G. Murray, "A computational model for TensorFlow: an introduction Proceedings of the 1st ACM SIGPLAN", International Workshop on Machine Learning and Programming Languages (2017) 1

[37] G. B. Goh, N. O. Hodas, \& A. Vishnu, "Deep learning for computational chemistry", Journal of computational chemistry 38 (2017) 1291.

[38] S. McArdle, S. Endo, A. Aspuru-Guzik, S. C. Benjamin, \& X. Yuan, "Quantum computational chemistry", Reviews of Modern Physics 92 (2020) 015003.

[39] L. Ling, F. Maohong, B. Wang, \& R. Zhang, "Application of computational chemistry in understanding the mechanisms of mercury removal technologies", a review Energy \& Environmental Science 8 (2015) 3109.

[40] D. L. Monego, M. Barcellos da Rosa, \& P. Cícero do Nascimento, "Applications of computational chemistry to the study of the antiradical activity of carotenoids", A review Food chemistry 217 (2017) 37.

[41] P. Gatt, R. Stranger, \& R. J. Pace, “ Application of computational chemistry to understanding the structure and mechanism of the Mn catalytic site in photosystem II", - a review Journal of Photochemistry and Photobiology B: Biology 104 (2011) 80.

[42] E. Soriano \& I. Fernandez, "Allenes and computational chemistry: from bonding situations to reaction mechanisms", Chemical Society Reviews 43 (2014) 3041.

[43] L. Ziheng, "Computational discovery of energy materials in the era of big data and machine learning", a critical review Materials Reports: Energy (2021) 100047.

[44] J. A. Keith, V. Vassilev-Galindo, B. Cheng, S. Chmiela, M. Gastegger, M. Klaus-Robert, \& A. Tkatchenko, "Combining machine learning and computational chemistry for predictive insights into chemical systems arXiv [preprint] (2021)", arXiv: (2102.06321).

[45] P. Abhik, A. Sarkar, S. Saha, A. Maji, P. Janah, T. M. Kumar, "Synthetic and computational efforts towards the development of peptidomimetics and small-molecule SARS-CoV 3CLpro inhibitors", a review Bioorganic \& Medicinal Chemistry (2021) 116301.

[46] T. T. Huong, N. V. Tran, A. K. Tieu, H. Zhu, H. Yu, \& D. T. Thi, "Computational Tribochemistry: A Review from Classical and Quantum Mechanics Studies", The Journal of Physical Chemistry 34 (2021) 1004.

[47] H. Lei, L. Bai, D. D. Dionysiou, Z. Wei, R. Spinney, C. Chu, L. Zhang, \& R. Xiao, "Applications of computational chemistry, artificial intelligence, and machine learning in aquatic chemistry research", Chemical Engineering Journal 56 (2021) 131810.

[48] H. L. Peña, D. A. Boateng, S. McPherson, \& K. M. Tibbetts, "Using computational chemistry to design pump-probe schemes for measuring radical cation dynamics", Physical Chemistry Chemical Physics 76 (2021) 205. 